\title{
Usage of QR code for Resource Planning and Utilization
}

\author{
J. Sowbakiyam ${ }^{1}$, E.Ramya ${ }^{2}$, Dr.Priyadharshini.M ${ }^{3}$ \\ Computer Science and Engineering, KPRIET, Coimbatore, TamilNadu, India ${ }^{1,2,3}$
}

\begin{abstract}
To solve the risk of counterfeiting and helping in revenue for goodwill of industry, usage of QR code for resource planning and utilization is followed. Many organizations are facing the problem of poor utilization of resources due to lack of planning and it leads to difficulty in achieving corporate organizational objectives. The work examines the need and scan a QR code by the android device where it directs to the data that displays resources used and planning measures. A convenient way of tracking and controlling process as it could be deployed on smart phones and other devices. It will reduce the worker's efforts to manually maintain the track of each item .Resource planning consume a desired quantity and excess inventory. This invention also comprises a system and storage. The handheld device automatically fetched an information from the server and it will produce manufacturing details. The objective of the proposed system is, the workers to maintain accurate machine details in analyzed manner and will reduce the process time.
\end{abstract}

Keywords: QR code Scanner, Resource planning and utilization management, QR code

\section{INTRODUCTION}

QR is short for Quick Response. QR codes are easiest way to providing an information to the consumer and allow them to view essential details. The reason why these QR codes are more useful than a standard barcode is that they can store and digitally present much more data, including URL links, text and Geo coordinates .The other key feature of QR Codes is that instead of requiring a chunky hand-held scanner to scan them, any Android mobile phones can scan them. QR based resource planning and utilization management is an android application which utilizes the QR code scanner in the mobile to record and maintain the raw material details.

\section{TYPES OF QR CODE}

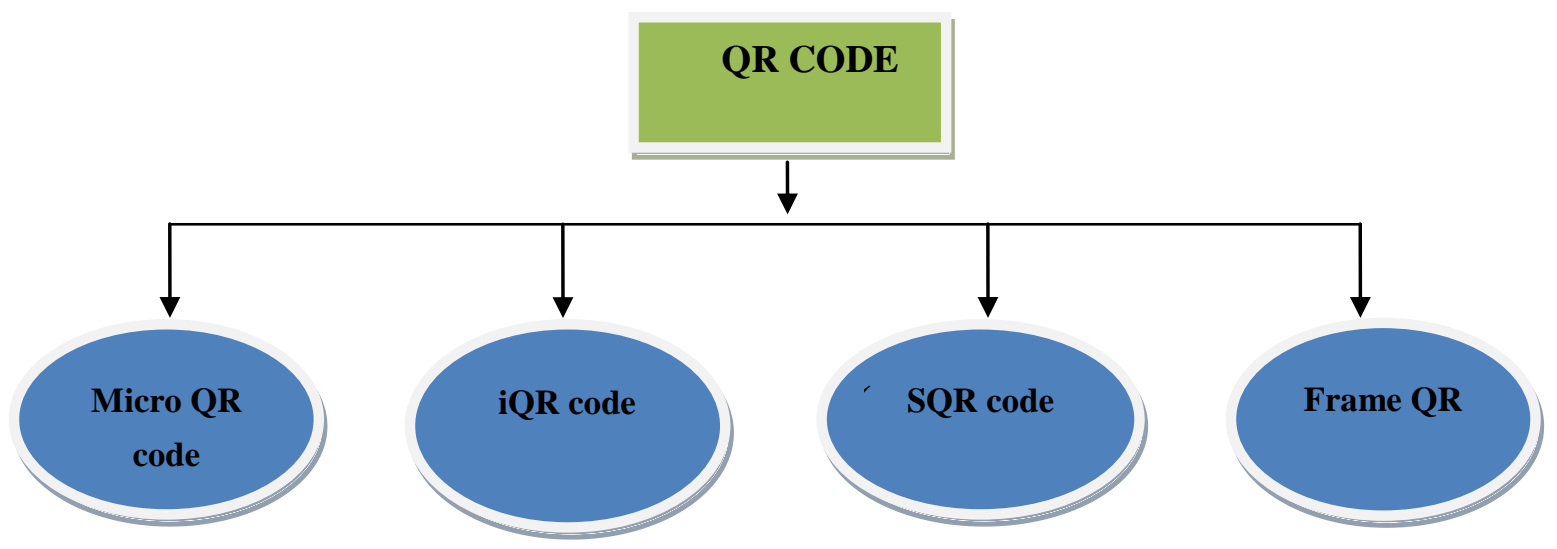

\section{$\checkmark \quad$ Micro QR code}

Micro QR code is a smaller version of the QR code standard for applications where symbol size is limited that is one position detection pattern, compared with regular $\mathrm{QR}$ code that require a certain amount of area because position detection patterns are located at three corners of the symbol. There are four different sizes or versions of Micro QR codes, the smallest is $11 \times 11$ modules and the largest can hold 35 numeric characters.

\section{$\checkmark \quad$ iQR code}

$\mathrm{iQR}$ Code is a easy way to read the data of any position and size. iQR code allows a wide size range of code from ones smaller than the Micro QR Code and traditional QR code to large ones that can store more data more than the other QR code does. iQR code can be printed as a rectangular code, turned-over code, black-and-white inversion code or dot pattern code as well, it allows a wide range of applications in various areas that is intended for situations where a 
Vol. 8, Issue 2, February 2019

rectangular barcode would otherwise be more appropriate, as such as cylindrical objects. The iQR codes can fit the same amount of information even in $30 \%$ of less space. There are 15 versions of rectangular codes and 61 versions of square iQR codes[5]. For squares, the minimum size is $9 \times 9$ modules and for rectangles have a minimum of $19 \times 5$ modules. The iQR codes add error correction level S, which can allow for 50\% error correction. iQR Codes have not yet been given an ISO specification, and only proprietary Denso Wave products can create or read the iQR codes.

\section{$\checkmark \quad$ SQR code}

Secure Quick Response code (SQR code) is a secure type for encrypting data in a QR code. This type of QR code contains a "private data" segment after the terminator instead of the specified filler bytes "ec 11".This private data segment must be deciphered with an encryption key. This SQR code can be used to store private informations and to manage company's internal information. This is the more secure type of QR code as it stores the data in encrypted form.

\section{$\checkmark \quad$ Frame QR}

Frame QR code is with a "canvas area" that can be flexibly used. In the center of this Frame code there is a canvas area where graphics, letters and more data can be flexibly arranged, making it possible to different layout for the code without losing the design of illustrations, images, etc[5]. The Model $1 Q R$ code is the older version of the specification. The Frame QR code is visually similar to the other types of model 2 codes, but lacks alignment patterns.

\section{APPLICATION DOMAINS}

\section{Product Tracking}

Use QR codes to track a products through mobile devices and any delay in delivering the consumer easily identify the product location. If there any document is missing or damaged shipments in real time using a single device and instantly document delivery information also obtained[6]. When circumstances warrant it, drivers can also use smart devices to verify the ID of parcel recipients. Instructing them about delivery address changes, delivery time restrictions, and required ID verification at the point of delivery. In courier service they use QR code to track and view an information about their products by scanning the code.

\section{Paytm}

Paytm is an online transaction service, which enables us to link our bank account to the application. Whenever we need a transaction to take place, we could scan the QR code or UPI pin and make a transaction. The amount will be credited from the bank account. This online transaction used in movie ticket booking, shopping, hotel booking and bus ticket booking.

\section{Medical Field}

Entry of the contents of prescription is made smoother through by scanning the QR codes generated on prescriptions.

\section{Admission control}

In admission venues scanning QR tickets at the entrance of attractions and shows using mobile device makes admission control possible even at venues where gate cannot be installed.

\section{Advertisement}

QR codes have become common in consumer advertising field. Now-a -days the QR code has become a focus for advertising strategy, since it provides a way to access a brand's website more quickly than by manually entering a URL. It continuously interest the prospects further down the conversion funnel with little delay or effort, bringing the viewer to the advertiser's website immediately, where a longer and more targeted sales pitch may lose the viewer's interest. After QR code read that contains an information about,

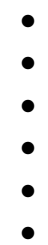

Product details

Contact details

Offer details

Event details

Competition details

$\bullet$

A coupon

\section{WORKING OF QR CODE SYSTEM}

QR Codes are 2-dimensional data, which results in having a square filled with data. Besides data, there are certain other identifiers that helps the code being read accurately. The most common QR Code type is the model 2, which is broken down into the following information identifiers: 

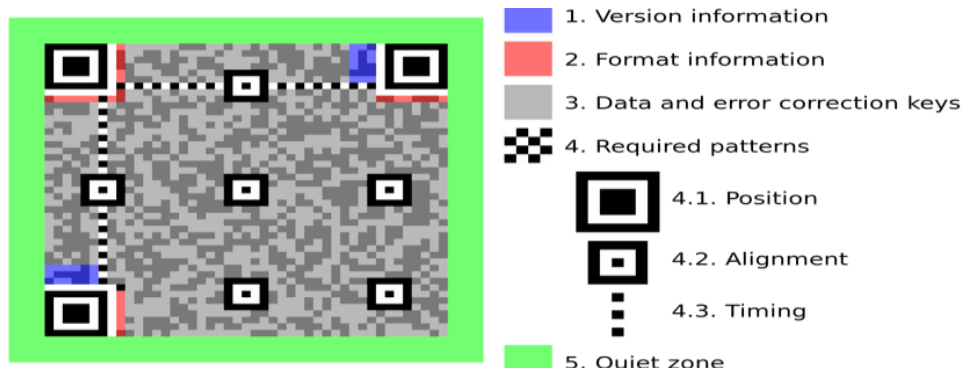

The format and the version information are important for the scanning device to know what kind of data to be expect. Meanwhile, the information can be slightly smeared or missing and still be readable. This depends on the error correction level being used when generating the code. The QR code can rotated and scanned however the user like. Upside down will still works. This is a courtesy of the position patterns the squares with dots in the middle that allow the code to be read from any direction that is of 360 degrees .Meanwhile, the alignment patterns are used to assist in the navigation of the larger set of codes and the timing of the code patterns are used to determine the size of modules. The quiet zone requires a margin of at least 4-module worth to it.

\section{SYSTEM ARCHITECTURE}

The user can generate a QR code for an invention machine to identify the used raw materials . After generating QR code adding records as the name suggests adds a new item to the database and each item is given a unique SKU. Generating QR code involves generation of QR Code which can be used to attach it to each item. Afterwards the generated QR code can then be printed by Label printer. Scanning QR code consists of scanning of QR code which are attached to each item and after the QR Code is scanned the item related information is shown on the screen.

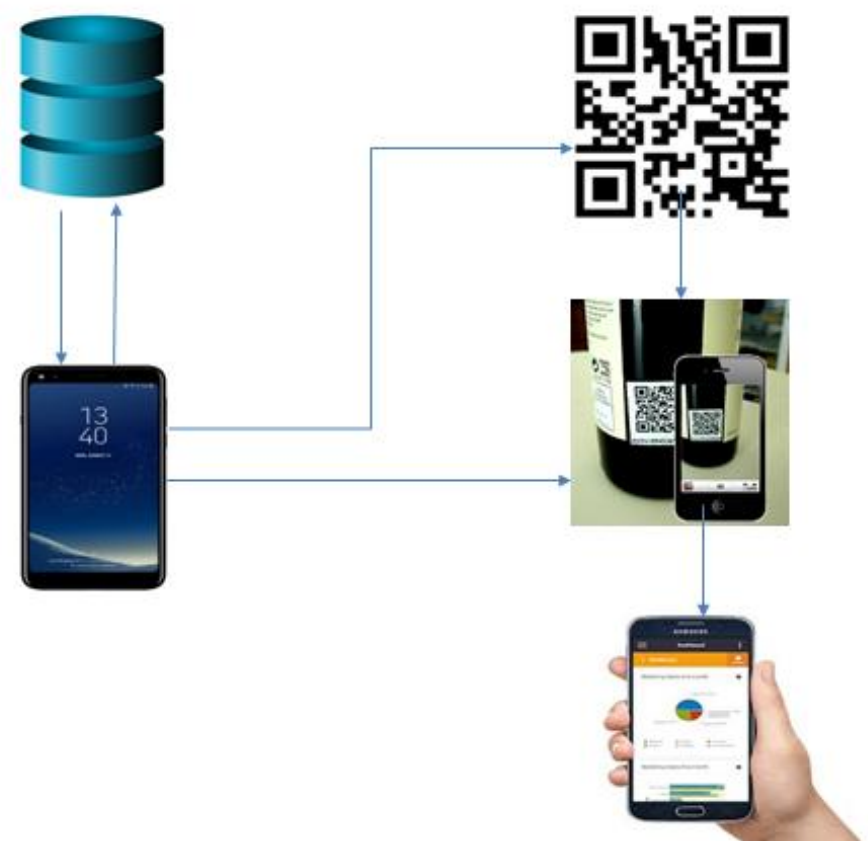

System Architecture

\section{CONCLUSION}

Thus a complete resource planning and utilization management system that can help the managers/workers/owners of the industry to maintain their raw materials using the QR code scanner. This process can reduce the worker's effort of manually maintaining the track of each machine and their headache of maintaining the register for the raw materials and stocks used since everything would be stored in the database. The development of this project will help the workers give accurate machine details and will reduce the process time in which workers doesn't need to manually verify or ask anyone about the information related to the machine and also will helps in resource planning and its utilization. 


\section{IJARCCE}

\section{REFERENCES}

[1]. https://www.irjet.net/archives/V4/i6/IRJET-V4I6506.pdf

[2]. Barcode based Student Attendance System, International Journal of Computer Applications(0975-8887), Volume 119 - No.2, June 2015.

[3]. http://www.explainthatstuff.com/how-data-matrix-codes-work.html.

[4]. https://dl.acm.org/citation.cfm?id=168

[5]. https://www.qrcode.com/en/codes/

[6]. https://www.scandit.com/types-barcodes-choosing-right-barcode/

[7]. http://goqr.me/api/

[8]. https://github.com/zxing/zxing

[9]. https://www.semantics3.com/blog/why-the-u-in-upc-doesnt-mean-universal-a1a675eea0ea

[10]. https://www.denso-wave.com/en/adcd/fundamental/2dcode/case/index.html

[11]. https://www.google.com/amp/s/thedigitalprojectmanager.com/12-project-management-software-resource-scheduling-tools/amp/

[12]. https://www.gocodes.com/resources-using-qr-codes-to-track-equipment-maintenance

[13]. https://www.google.com/url?sa=t\&source=web\&rct=j\&url=https://ahsri.uow.edu.au/content/groups/public/\%40web/\%40chsd/documents/doc/u ow244012.pdf\&ved=2ahUKEwiL4fiUubzfAhWBV30KHUKDBlgQFjAEegQIBhAB\&usg=AOvVaw2jSnQKdwSrrE27KBIIESY7\&cshid=154 5791448068

[14]. http://technav.ieee.org/tag/5873/enterprise-resource-planning

[15]. http://scholar.google.co.in/scholar?q=resource+planning++and+utilization+ieee+papers\&hl=en\&as_sdt=0\&as_vis=1\&oi=scholart 